\title{
Genome-wide DNA methylation analysis of heavy cannabis exposure in a New Zealand longitudinal cohort
}

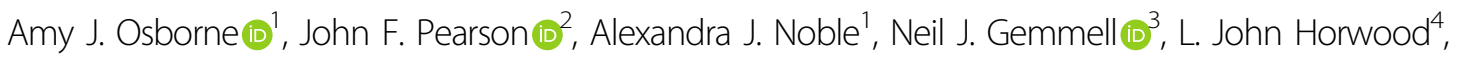

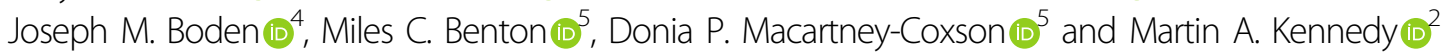

\begin{abstract}
Cannabis use is of increasing public health interest globally. Here we examined the effect of heavy cannabis use, with and without tobacco, on genome-wide DNA methylation in a longitudinal birth cohort (Christchurch Health and Development Study, CHDS). A total of 48 heavy cannabis users were selected from the CHDS cohort, on the basis of their adult exposure to cannabis and tobacco, and DNA methylation assessed from whole blood samples, collected at approximately age 28. Methylation in heavy cannabis users was assessed, relative to non-users ( $n=48$ controls) via the Illumina Infinium ${ }^{\circledR}$ MethylationEPIC BeadChip. We found the most differentially methylated sites in cannabis with tobacco users were in the $A H R R$ and F2RL3 genes, replicating previous studies on the effects of tobacco. Cannabis-only users had no evidence of differential methylation in these genes, or at any other loci at the epigenome-wide significance level $\left(P<10^{-7}\right)$. However, there were 521 sites differentially methylated at $P<0.001$ which were enriched for genes involved in neuronal signalling (glutamatergic synapse and long-term potentiation) and cardiomyopathy. Further, the most differentially methylated loci were associated with genes with reported roles in brain function (e.g. TMEM190, MUC3L, CDC20 and SP9). We conclude that the effects of cannabis use on the mature human blood methylome differ from, and are less pronounced than, the effects of tobacco use, and that larger sample sizes are required to investigate this further.
\end{abstract}

\section{Introduction}

Cannabis use is an important global public health issue, and a growing topic of controversy and debate ${ }^{1,2}$. It is the most widely used illicit psychoactive substance in the world ${ }^{3}$, and the potential medicinal and therapeutic benefits of cannabis and its main active ingredients tetrahydrocannabinol (THC) and cannabidiol (CBD) are gaining interest ${ }^{4-6}$. There is strong evidence to suggest that the heavy and prolonged use of cannabis may be associated with increased risk of adverse outcomes in a

Correspondence: Amy J. Osborne (amy.osborne@canterbury.ac.nz) or Martin A. Kennedy (martin.kennedy@otago.ac.nz)

${ }^{1}$ School of Biological Sciences, University of Canterbury, Christchurch 8041, New Zealand

${ }^{2}$ Department of Pathology and Biomedical Science, University of Otago

Christchurch, Christchurch 8011, New Zealand

Full list of author information is available at the end of the article

These authors contributed equally: Amy J. Osborne, John F. Pearson number of areas, including mental health (psychosis ${ }^{7-9}$, schizophrenia $^{10,11}$, depression ${ }^{12,13}$ ) and illicit drug abuse ${ }^{14}$.

Drug metabolism, drug response and drug addiction have known genetic components ${ }^{15}$, and multiple genome-wide association studies (GWAS) have identified genes and allelic variants that are likely contributors to substance use disorders $^{16,17}$. There are aspects of cannabis use disorder that are heritable ${ }^{18-21}$, and several candidate loci for complex phenotypes such as lifetime cannabis use have recently been identified $^{3,22}$ that explain a proportion of the variance in cannabis use heritability. Complex phenotypes like these are influenced by multiple loci, each of which usually has a small individual effect size ${ }^{23}$, and such loci are frequently located in non-coding regions of the genome ${ }^{24,25}$, making their biological role difficult to elucidate.

Epigenetic mechanisms are involved in the interaction between the genome and environment; they respond to

\section{(c) The Author(s) 2020}

(c) (i) Open Access This article is licensed under a Creative Commons Attribution 4.0 International License, which permits use, sharing, adaptation, distribution and reproduction cc) in any medium or format, as long as you give appropriate credit to the original author(s) and the source, provide a link to the Creative Commons license, and indicate if changes were made. The images or other third party material in this article are included in the article's Creative Commons license, unless indicated otherwise in a credit line to the material. If material is not included in the article's Creative Commons license and your intended use is not permitted by statutory regulation or exceeds the permitted use, you will need to obtain permission directly from the copyright holder. To view a copy of this license, visit http://creativecommons.org/licenses/by/4.0/. 
changes in environmental stimuli (such as diet, exercise, drugs), and act to alter chromatin structure and thus regulate gene expression ${ }^{26}$. Epigenetic modifications, such as DNA methylation, contribute to complex traits and diseases ${ }^{27,28}$. Methylation of cytosine residues within CpG dinucleotides is an important mechanism of variation and regulation in the genome ${ }^{29-32}$. Cytosine methylation, particularly in the promoter region of genes, is often associated with a decrease in transcription ${ }^{33}$, and DNA methylation in the first intron and gene expression is correlated and conserved across tissues and vertebrate species $^{34}$. Furthermore, modulation of methylation at CpG sites within the human genome can result in an epigenetic pattern that is specific to individual environmental exposures, and these may contribute to disease $^{26,35-37}$. For example, environmental factors such as drugs, alcohol, stress, nutrition, bacterial infection, and exercise $^{36,38-41}$ have been associated with methylation changes. A number of these methylation changes have been shown to endure and induce lasting biological changes $^{36}$, whereas others are dynamic and transient. For example, alcohol consumption affects genome-wide methylation patterns in a severity-dependent manner ${ }^{42}$ and some of these changes revert upon abstinence from alcohol consumption ${ }^{43}$. A similar observation is reported for former tobacco smokers, with DNA methylation changes after cessation eventually reaching levels close to those who had never smoked tobacco ${ }^{44}$. Thus, DNA methylation can be indicative of a particular environmental exposure, shed light on the dynamic interaction between the environment and the genome, and provide new insights in to the biological response.

Recreational drug use (an environmental stimulus) has been associated with adverse mental health outcomes, particularly in youth ${ }^{45-49}$, and epigenetics may play a role in mediating the biology involved. Therefore, we sought to determine whether regular cannabis users displayed differential cytosine methylation compared with noncannabis users. Cannabis users in this study are participants from the Christchurch Health and Development Study (CHDS), a longitudinal study of a birth cohort of 1265 children born in 1977 in Christchurch, New Zealand. Users often consume cannabis in combination with tobacco. Unusually, the CHDS cohort contains a subset of cannabis users who have never consumed tobacco, thus enabling an investigation of the specific effects of cannabis consumption, in isolation, on DNA methylation in the human genome.

\section{Methods}

\section{Cohort and study design}

The Christchurch Health and Development Study includes individuals who have been studied on 24 occasions from birth to the age of $40(n=987$ at age 30 , with blood collected at approximately age 28). In the early 1990s, research began into the initiation and consequences of cannabis use amongst CHDS participants; cannabis use was assessed prospectively over the period up to the collection of DNA ${ }^{11-14,48-54}$. A subset of $n=96$ participants for whom a blood sample was available are included in the current study. Cases (regular cannabis users, $n=48)$ were matched with controls $(n=48)$ for sex ( $n=37$ male, $n=11$ female each group, for additional information see Supplementary Table 1). Case participants were partitioned into two subsets: one that contained cannabis-only users (who had never consumed tobacco, "cannabis-only", $n=24 \quad[n=21$ male, $n=3$ female]), and one that contained cannabis users who also consumed tobacco ("cannabis with tobacco", $n=24$ [ $n=$ 16 male, $n=8$ female]) and were selected on the basis that they either met DSM-IV ${ }^{55}$ diagnostic criteria for cannabis dependence, or had reported using cannabis on a daily basis for a minimum of three years, prior to age 28 . Of the 48 cannabis users, 6 participants had ceased cannabis use by 28 years of age, however, still met the diagnostic criteria for cannabis dependence. Mode of cannabis consumption was via smoking, for all participants. The median duration of regular use was 9 years (range 3-14 years). Control participants had never used cannabis or tobacco. In addition, comprehensive single nucleotide polymorphism (SNP) data was available for all participants $^{56}$. All aspects of the study were approved by the Southern Health and Disability Ethics Committee, under application number CTB/04/11/234/AM10 "Collection of DNA in the Christchurch Health and Development Study", and the CHDS ethics approval covering collection of cannabis use: "16/STH/188/AM03 The Christchurch Health and Development Study 40 Year Follow-up".

\section{DNA extraction and methylation arrays}

DNA was extracted from whole blood using the KingFisher Flex System (Thermo Scientific, Waltham, MA, USA), as per the published protocols. DNA was quantified via NanoDrop ${ }^{\mathrm{TM}}$ (Thermo Scientific, Waltham, MA, USA) and standardised to $100 \mathrm{ng} / \mu \mathrm{l}$. Equimolar amounts were shipped to the Australian Genomics Research Facility (AGRF, Melbourne, VIC, Australia) for analysis with the Infinium ${ }^{\circledast}$ MethylationEPIC BeadChip (Illumina, San Diego, CA, USA).

\section{Bioinformatics and statistics}

All analysis was carried out using R (Version 3.5.2 ${ }^{57}$ ). Prior to normalisation, quality control was performed on the raw data. Firstly, sex chromosomes and 150 failed probes (detection $P$ value $>0.01$ in at least $50 \%$ of samples) were excluded from analysis. Furthermore, potentially problematic CpGs with adjacent SNVs, or that did not map to a unique location in the genome ${ }^{58}$, were also 
excluded, leaving 700,296 CpG sites for further analysis. The raw data were then normalised with the NOOB procedure in the minfi package ${ }^{59}$ (Supplementary Fig. 1). Normalisation was checked by visual inspection of intensity densities and the first two components from Multi-Dimensional Scaling of the 5000 most variable CpG sites (Supplementary Figs. 2 and 3). The proportions of cell types $(\mathrm{CD} 4+, \mathrm{CD} 8+\mathrm{T}$ cells, natural killer, B cells, monocytes and granulocytes) in each sample were estimated with the Flow.Sorted.Blood package ${ }^{60}$. Linear models were fitted to the methylated/unmethylated or M ratios using limma ${ }^{61}$. Separate models were fitted for cannabis-only vs. controls, and cannabis plus tobacco users vs. controls. Both models contained covariates for sex (bivariate), socioeconomic status (three levels), batch (bivariate), population stratification (four principal components from 5000 most variable SNPs) and cell type (five continuous). $\beta$ values were calculated, defined as the ratio of the methylated probe intensity $(\mathrm{M}) /$ the sum of the overall intensity of both the unmethylated probe $(\mathrm{U})+$ methylated probe $(\mathrm{M}) . P$ values were adjusted for multiple testing with the Benjamini and Hochberg method and assessed for genomic inflation with bacon ${ }^{62}$. Differentially methylated $\mathrm{CpG}$ sites that were intergenic were matched to the nearest neighbouring genes in Hg19 using GRanges $^{63}$, and the official gene symbols of all significantly differentially methylated CpG sites (nominal $P<0.001$ ) in cannabis-only users were tested for enrichment in KEGG 2019 human pathways with EnrichR ${ }^{64}$.

\section{Results}

\section{Data normalisation}

Modelled effects showed no indication of genomic inflation with $\lambda=1.04$ for cannabis-only users (Supplementary Fig. 4a) and $\lambda=0.855$ for cannabis with tobacco users (Supplementary Fig. 4b), versus controls. These were confirmed with bacon for cannabis-only (inflation = 0.98 , bias $=0.044$ ) and cannabis with tobacco users (inflation $=0.91$, bias $=0.19$ ). Inflation values $<1$ suggest that the results may be conservative.

Cannabis with tobacco users had a significantly lower estimated proportion of natural killer cells than controls $(1.8 \%, 0.4-3.2 \%, P<0.014)$ with no other proportions differing significantly. After adjusting for multiple comparisons this was not significant $(P=0.08)$, however, we note that it is consistent with other findings that NK-cells are suppressed in the plasma of tobacco smokers ${ }^{65,66}$.

\section{Differential methylation}

The most differentially methylated CpG sites for cannabis users relative to controls differed in the absence (Table 1) and presence (Table 2) of tobacco smoking. Five individual $\mathrm{CPG}$ sites were significantly differentially methylated ( $P$ adjusted $<0.008$ ) between cannabis users

Table 1 Top 15 differentially methylated CpG sites in cannabis-only users vs controls.

\begin{tabular}{|c|c|c|c|c|c|c|c|c|}
\hline CpG & Gene & Location & $\begin{array}{l}\text { Distance } \\
\text { (bp) }\end{array}$ & $\begin{array}{l}\text { Cannabis } \\
\beta_{U}\end{array}$ & $\begin{array}{l}\text { Control } \\
\beta_{C}\end{array}$ & $\begin{array}{l}\text { Difference } \\
\beta_{U}-\beta_{C}\end{array}$ & $\begin{array}{l}P \text { value } \\
\text { Nominal }\end{array}$ & $\begin{array}{l}P \text { value } \\
\text { Adjusted }\end{array}$ \\
\hline cg12803068 & MYO1G & Intron & & 0.8 & 0.71 & 0.1 & $6.30 \mathrm{E}-07$ & 0.4 \\
\hline cg02234936 & ARHGEF1 & Intron & & 0.14 & 0.13 & 0.01 & $1.10 \mathrm{E}-06$ & 0.4 \\
\hline cg01695406 & TMEM190 & Intron & & 0.82 & 0.77 & 0.05 & $3.00 \mathrm{E}-06$ & 0.6 \\
\hline cg24875484 & MUCL3 & Intron & & 0.1 & 0.09 & 0.01 & $3.90 \mathrm{E}-06$ & 0.6 \\
\hline cg05009104 & MYO1G & Intron & & 0.79 & 0.74 & 0.05 & $5.90 \mathrm{E}-06$ & 0.6 \\
\hline cg00470351 & CDC20 & Exon & & 0.4 & 0.38 & 0.02 & $6.10 E-06$ & 0.6 \\
\hline cg24060040 & DUS3L & Upstream & 11,018 & 0.11 & 0.08 & 0.03 & $6.30 \mathrm{E}-06$ & 0.6 \\
\hline cg12322720 & FOXB1 & Downstream & 150,921 & 0.58 & 0.52 & 0.06 & $8.90 \mathrm{E}-06$ & 0.7 \\
\hline cg16746471 & KIAA1324L & Promoter & 374 & 0.1 & 0.08 & 0.02 & $1.10 \mathrm{E}-05$ & 0.7 \\
\hline cg04180046 & MYO1G & Intron & & 0.56 & 0.52 & 0.04 & $1.20 \mathrm{E}-05$ & 0.7 \\
\hline cg06955687 & DDX25 & Downstream & 28,769 & 0.74 & 0.7 & 0.04 & $1.20 \mathrm{E}-05$ & 0.7 \\
\hline ch.22.707049R & TNRC6B & Downstream & 159,737 & 0.06 & 0.04 & 0.01 & 1.30E-05 & 0.7 \\
\hline cg09344183 & SP9 & Downstream & 5964 & 0.06 & 0.05 & 0.01 & 1.40E-05 & 0.7 \\
\hline cg06693983 & TMEM190 & Exon & & 0.84 & 0.76 & 0.08 & $1.40 \mathrm{E}-05$ & 0.7 \\
\hline cg26069230 & ADAP2 & Exon & & 0.16 & 0.14 & 0.01 & $1.50 \mathrm{E}-05$ & 0.7 \\
\hline
\end{tabular}

Beta values with $P$ values, nominal and adjusted by the Benjamini and Hochberg method. Locations are relative to hg19 with gene names for overlapping genes or nearest $5^{\prime}$ gene with distance to the $5^{\prime}$ end shown. 
Table 2 Top 15 differentially methylated CpG sites in cannabis with tobacco users vs controls.

\begin{tabular}{|c|c|c|c|c|c|c|c|c|}
\hline CpG & Gene & Location & $\begin{array}{l}\text { Distance } \\
\text { (bp) }\end{array}$ & $\begin{array}{l}\text { Cannabis } \\
\beta_{U}\end{array}$ & $\begin{array}{l}\text { Control } \\
\beta_{C}\end{array}$ & $\begin{array}{l}\text { Difference } \\
\beta_{U}-\beta_{C}\end{array}$ & $\begin{array}{l}P \text { value } \\
\text { Nominal }\end{array}$ & $\begin{array}{l}P \text { value } \\
\text { Adjusted }\end{array}$ \\
\hline cg05575921 & AHRR & Intron & & 0.66 & 0.89 & -0.24 & $1.40 \mathrm{E}-11$ & 0.00001 \\
\hline cg21566642 & ALPG & Downstream & 13,109 & 0.44 & 0.62 & -0.17 & $9.90 \mathrm{E}-11$ & 0.00003 \\
\hline cg03636183 & F2RL3 & Exon & & 0.59 & 0.68 & -0.09 & $2.60 \mathrm{E}-09$ & 0.0006 \\
\hline cg01940273 & ALPG & Downstream & 13,382 & 0.53 & 0.63 & -0.09 & $3.60 \mathrm{E}-08$ & 0.00636 \\
\hline cg17739917 & RARA & Intron & & 0.37 & 0.47 & -0.1 & $5.60 \mathrm{E}-08$ & 0.00783 \\
\hline cg01541424 & LINC02393 & Upstream & 491,508 & 0.17 & 0.13 & 0.04 & $6.30 \mathrm{E}-07$ & 0.07 \\
\hline cg12828729 & TIFAB & Upstream & 35,880 & 0.56 & 0.5 & 0.06 & $7.10 \mathrm{E}-07$ & 0.07 \\
\hline cg10148067 & MTFR1 & Upstream & 3928 & 0.91 & 0.88 & 0.02 & $7.70 \mathrm{E}-07$ & 0.07 \\
\hline cg14391737 & PRSS23 & Intron & & 0.36 & 0.42 & -0.06 & $9.60 \mathrm{E}-07$ & 0.07 \\
\hline cg07219494 & TENM2 & Upstream & 303,359 & 0.7 & 0.75 & -0.05 & $1.40 \mathrm{E}-06$ & 0.1 \\
\hline cg05723029 & $\mathrm{PIEZO2}$ & Intron & & 0.83 & 0.79 & 0.05 & $1.50 \mathrm{E}-06$ & 0.1 \\
\hline cg03329539 & ALPG & Downstream & 11,777 & 0.36 & 0.41 & -0.05 & $3.20 \mathrm{E}-06$ & 0.2 \\
\hline cg24994593 & LDLRAD3 & Intron & & 0.9 & 0.89 & 0.02 & $4.20 \mathrm{E}-06$ & 0.2 \\
\hline cg25009999 & LINC01168 & Downstream & 14,152 & 0.93 & 0.92 & 0.01 & $5.60 \mathrm{E}-06$ & 0.3 \\
\hline cg13957017 & TTLL6 & Intron & & 0.72 & 0.69 & 0.03 & $7.30 \mathrm{E}-06$ & 0.3 \\
\hline
\end{tabular}

Beta values with $P$ values, nominal and adjusted by the Benjamini and Hochberg method. Locations are relative to hg19 with gene names for overlapping genes or nearest $5^{\prime}$ gene with distance to the $5^{\prime}$ end shown.

and controls when cannabis with tobacco was used (Table 2 and Fig. 1). The top CpG sites in the AHRR, ALPG and F2RL3 genes (Table 2) are consistent with previous studies on tobacco use without cannabis (e.g. refs. ${ }^{44,67-69}$ ), and cg17739917 is in the same CpG-island as other CpGs previously shown to be hypomethylated in response to tobacco $^{70}$. Cannabis-only users showed no CpG sites differentially methylated after correction for multiple testing (Table 1 and Fig. 2), however, the most differentially methylated site was hypermethylation of cg12803068 in the gene $M Y O 1 G$, which has been reported to be hypermethylated in response to tobacco use ${ }^{67}$. We identified 28 genes with multiple (two or more) differentially methylated CpG probes (Supplementary Table 2). Of these 28 genes, 25 have all sites hypermethylated, one has two sites hypomethylated, two have one hypermethylated and one hypomethylated probe.

To describe the data we chose a nominal $P$ value of 0.001 , and observed that both cannabis-only and cannabis with tobacco users showed relatively higher rates of hypermethylation than hypomethylation compared with controls and that the distribution of these CpG sites was similar with respect to annotated genomic features (Table 3). Four CpG sites overlapped between the cannabis-only and cannabis with tobacco users analyses; two were hypermethylated; cg02514528, in the promoter of $M A R C 2$, and cg27405731 in CUX1, and one, cg26542660 in the promoter of CEP135, was hypomethylated in comparison to controls. The second most differentially methylated site (ranked by $P$ value) in cannabis-only users was cg02234936 which maps to ARHGEF1; this was hypermethylated in the cannabis with tobacco users.

\section{Pathway enrichment analyses}

We then took the genes containing differentially methylated $\mathrm{CpG}$ sites at $P<0.001$ for the cannabis-only group, or the closest gene where that $\mathrm{CpG}$ was intergenic (Supplementary Table 3) and compared them with human KEGG pathways using Enrichr. The hypermethylated CpG sites $(n=420)$ showed enrichment in the arrhythmogenic right ventricular cardiomyopathy, long-term potentiation, cAMP signalling, adrenergic signalling in cardiomyocytes, glutamatergic synapse, hypertrophic cardiomyopathy, dilated cardiomyopathy and nicotine addiction pathways at an adjusted $P<0.05$ (Fig. 3). Enrichment analysis of hypomethylated loci $(n=101)$ in cannabis-only users did not identify any KEGG pathways at or near adjusted significance $(P>0.05$, Fig. 4). We further submitted all differentially methylated CpG sites (hyper and hypomethyated) at a nominal $P<0.001$ to Enrichr, revealing significant enrichment for genes involved in the glutamatergic synapse (adjusted $P=0.012$ ), arrhythmogenic right 

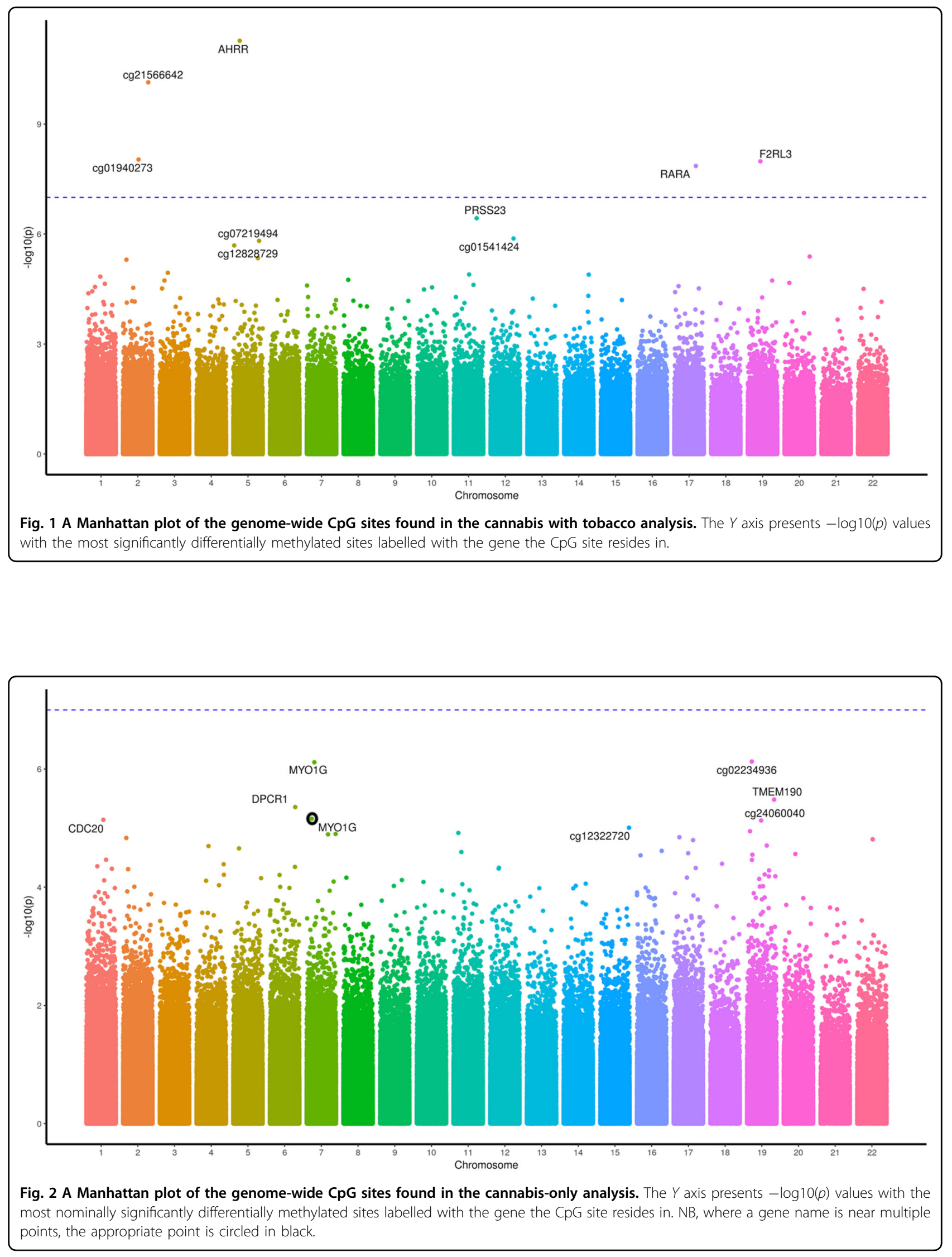
Table 3 Summary of CpG sites from cannabis-only and cannabis with tobacco users vs. non-users.

\begin{tabular}{|c|c|c|c|c|c|}
\hline & Cannabis-only & & Tobacco + Cannabis & & Both \\
\hline Differentially methylated loci $(F W E R=0.05)$ & 0 & & 6 & & \\
\hline \multicolumn{6}{|l|}{ Differentially methylated loci $(P<0.001)$} \\
\hline Total & 521 & & 533 & & \\
\hline Hypermethylated & 420 & $80.6 \%$ & 403 & $75.6 \%$ & 2 \\
\hline Hypomethylated & 101 & $19.4 \%$ & 130 & $24.4 \%$ & 1 \\
\hline Hyper (cannabis) Hypo (cannabis + tobacco) & & & & & 1 \\
\hline \multicolumn{6}{|l|}{ Location } \\
\hline Intron & 216 & $41.5 \%$ & 264 & $49.5 \%$ & \\
\hline Exon & 97 & $18.6 \%$ & 65 & $12.2 \%$ & \\
\hline Exon Boundary & 0 & & 0 & & \\
\hline Promoter & 89 & $17.1 \%$ & 60 & $11.3 \%$ & \\
\hline $3^{\prime}$ UTR & 3 & $0.6 \%$ & 1 & $0.2 \%$ & \\
\hline $5^{\prime}$ UTR & 0 & & 0 & & \\
\hline 3' (downstream) & 62 & $11.9 \%$ & 76 & $14.3 \%$ & \\
\hline 5' (upstream) & 54 & $10.4 \%$ & 67 & $12.6 \%$ & \\
\hline
\end{tabular}

Counts of significant sites at $P=0.001$ and at a Benjamini and Hochberg adjusted $P<0.05$. 'Both' indicates the number of $C p G$ sites of each type that are present and shared across both analyses.

FWER family-wise error rate.

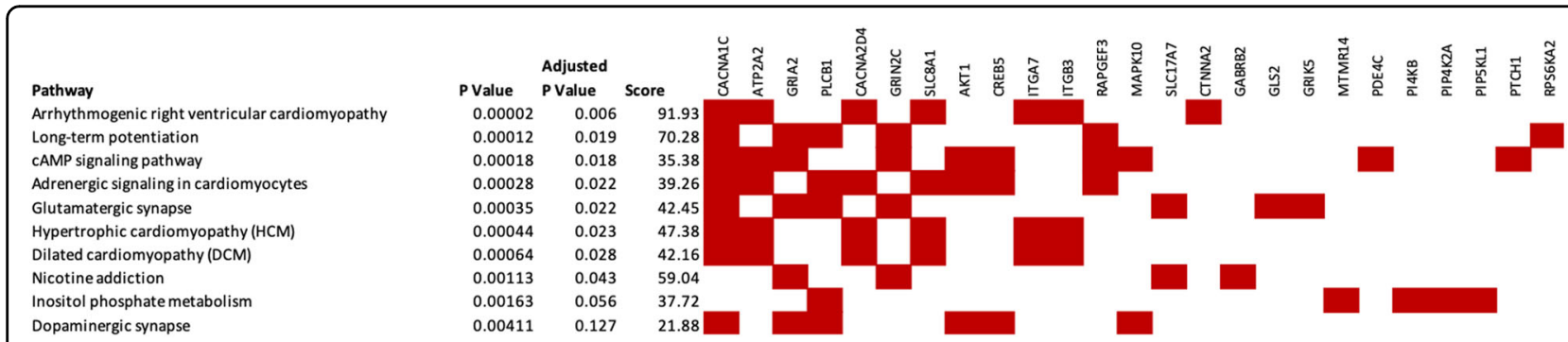

Fig. 3 Genetic networks enriched within the hypermethylated CpG sites identified in the cannabis-only analysis. Pathways from KEGG 2019. Genes shown by filled cells are hypermethylated in cannabis-only users and included in named pathway.

ventricular cardiomyopathy (adjusted $P=0.011$ ) and longterm potentiation pathways (adjusted $P=0.039$ ) (Fig. 5).

\section{Discussion}

Many countries have recently adopted, or are considering, lenient polices regarding the personal use of cannabis $^{71-73}$. This approach is supported by the evidence that the prohibition of cannabis can be harmful ${ }^{53}$. Further, the therapeutic benefits of cannabis are gaining traction, most recently as an opioid replacement therapy ${ }^{74}$. However, previous studies, including analyses of the CHDS cohort, have reported an association between cannabis use and poor health outcomes, particularly in youth ${ }^{75,76}$. Epigenetic mechanisms, including DNA methylation, provide the interface between the environment (e.g. cannabis exposure) and genome. Therefore, we investigated whether changes in an epigenetic mark, DNA methylation, were altered in cannabis users, versus controls, a comparison made possible by the deep phenotyping of the CHDS cohort with respect to cannabis use, and the fact that the widespread practice of mulling or mixing cannabis with tobacco, is not common in New Zealand.

Consistent with previous reports of tobacco exposure, we observed greatest differential methylation in cannabis with tobacco users in the $A H R R$ and $F 2 R L 3$ genes $^{44,67-69}$. These changes, however, were not apparent in the cannabis-only data. Only two nominally significantly differentially methylated $(P<0.05)$ CpG sites were observed in both the cannabis-only and cannabis with tobacco 


\section{Pathway}

Endocytosis

Glutamatergic synapse

Thyroid hormone signaling pathway
Human papillomavirus infection

Selenocompound metabolism

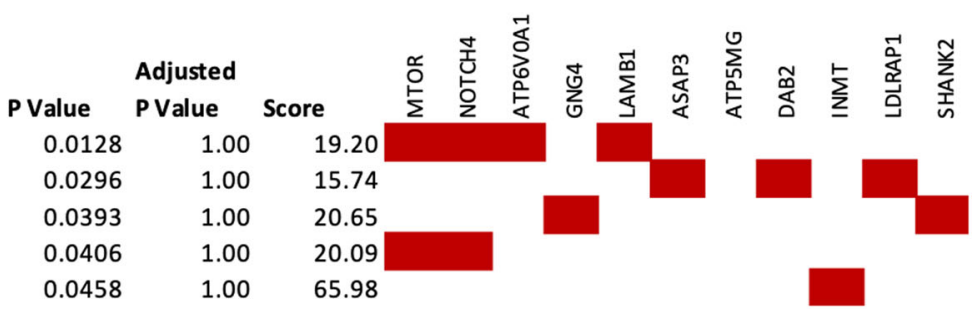

Fig. 4 Genetic networks enriched within the hypomethylated CpG sites identified in the cannabis-only users. Pathways from KEGG 2019. Genes shown by filled cells are hypomethylated in cannabis-only users and included in named pathway.

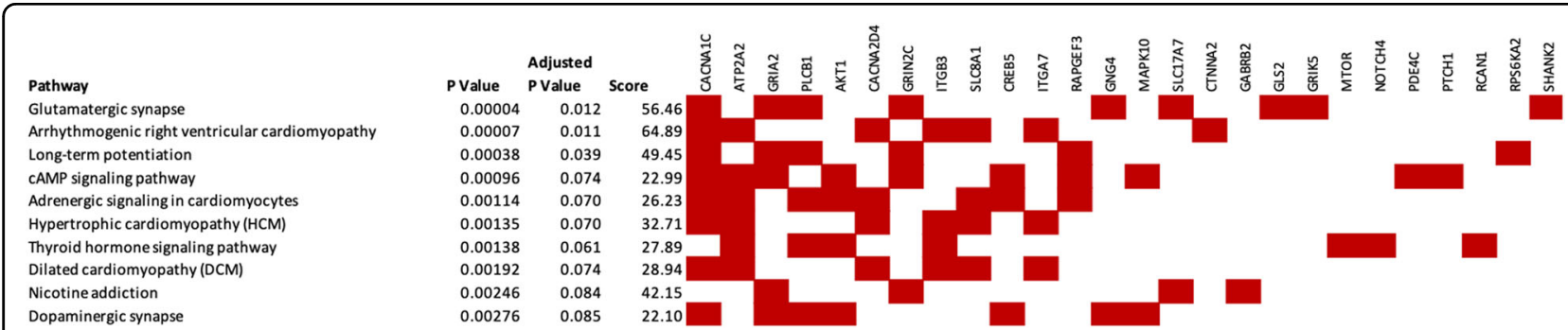

Fig. 5 Genetic networks enriched within the hypomethylated or hypermethylated CpG sites identified in the cannabis-only users. Pathways from KEGG 2019. Genes shown by filled cells are hypomethylated in cannabis-only users and included in named pathway.

analyses. This suggests that tobacco may have a more pronounced effect on DNA methylation and/or dominates any effects of cannabis on the human blood methylome, and that caution should be taken when interpreting similar cannabis exposure studies which do not, or cannot, exclude tobacco smokers. Interestingly, the two nominally significant $\mathrm{CpG}$ sites $(P<0.05)$ that overlap between the cannabis-only and the cannabis with tobacco data are located within the MARC2 and CUX1 genes, which both have reported roles in brain function; a SNP in MARC2 has been provisionally associated with the biological response to antipsychotic therapy in schizophrenia patients ${ }^{77}$, and the CUX1 gene has an established role in neural development ${ }^{78}$.

Cannabis affects the brain, leading to perceptual alterations, euphoria and relaxation ${ }^{18}$, and prolonged use is associated with mood disorders, including adult psy$\operatorname{chosis}^{7,8,49,79,80}$, mania $^{13}$, and depression ${ }^{12}$. We did not detect significantly differentially methylated loci associated with exclusive cannabis use at the epigenome-wide level. However, an assessment of those top loci reaching nominal significance $(P<0.05)$ identified $C p G$ sites within genes involved in brain function and mood disorders, including $M U C 3 L^{81,82}, C D C 20^{83}, D U S 3 L^{84}, T M E M 190^{85}$, FOXB1 $1^{86-88}, \quad K I A A 1324 L / G R M 3^{82,89-94}, \quad D D X 25^{81,95,96}$, TNRC6B ${ }^{97,98}$ and $S P 9^{99}$.

Pathway enrichment revealed that differential methylation in cannabis-only users was over-represented in genes associated with neural signalling and cardiomyopathies. This is consistent with the literature which raises clinical concerns around cardiac complications potentially associated with cannabis use $\mathrm{e}^{100-103}$. The enrichment of genes associated with neural signalling pathways is also consistent with the literature, including previous analyses of the CHDS cohort, which report associations between cannabis exposure and brain related biology such as mood disorders ${ }^{7,12,48,49,51-54,104,105}$. Our study was limited by sample size, achieving $\sim 10 \%$ power at $P=10^{-7}$ to detect the largest standardised effect size found. However, while we have not implicated any gene at the genome-wide significance level with respect to differential methylation associated with cannabis-only exposure, our data are suggestive of a role for DNA methylation in the biological response to cannabis, a possibility which definitely warrants further investigations in larger cohorts.

In summary, while tobacco use has declined on the back of state-sponsored cessation programmes ${ }^{106}$, rates of cannabis use remain high in New Zealand and globally, and might be predicted to increase further with the decriminalisation or legalisation of cannabis use for therapeutic and/or recreational purposes ${ }^{107}$. Therefore, analysis of the potential effects of cannabis (an environmental stimuli) on DNA methylation, an epigenetic mechanism, is timely. Our data are suggestive of a role for DNA methylation in the biological response to cannabis, significantly contributes to the growing literature studying 
the biological effects of heavy cannabis use, and highlights areas of further analysis in particular with respect to the epigenome.

\section{Acknowledgements}

The authors thank Allison Miller for technical assistance. CHDS, University of Otago Division of Health Sciences Collaborative Postdoctoral Fellowship to A.J.O., University of Otago Research Grant to M.A.K., The Carney Centre for Pharmacogenomics. CHDS was funded by the Health Research Council of New Zealand (Programme Grant 16/600) and the Canterbury Medical Research Foundation.

\section{Author details}

${ }^{1}$ School of Biological Sciences, University of Canterbury, Christchurch 8041, New Zealand. ${ }^{2}$ Department of Pathology and Biomedical Science, University of Otago Christchurch, Christchurch 8011, New Zealand. ${ }^{3}$ Department of Anatomy, Otago School of Medical Sciences, University of Otago, Dunedin 9054, New Zealand. ${ }^{4}$ Department of Psychological Medicine, University of Otago Christchurch, Christchurch 8011, New Zealand. ${ }^{5}$ Human Genomics, Institute of Environmental Science and Research, Kenepuru Science Centre, Porirua 5240, New Zealand

\section{Conflict of interest}

The authors declare that they have no conflict of interest.

\section{Publisher's note}

Springer Nature remains neutral with regard to jurisdictional claims in published maps and institutional affiliations.

Supplementary Information accompanies this paper at (https://doi.org/ 10.1038/s41398-020-0800-3).

Received: 4 February 2020 Revised: 11 March 2020 Accepted: 20 March 2020

Published online: 22 April 2020

\section{References}

1. Cressey, D. The cannabis experiment. Nature 524, 280-283 (2015).

2. Goldman, D. America's cannabis experiment. JAMA Psychiatry 72, 969-970 (2015).

3. Stringer, S. et al. Genome-wide association study of lifetime cannabis use based on a large meta-analytic sample of 32330 subjects from the International Cannabis Consortium. Transl. Psychiatry 6, e769 (2016).

4. Robson, P. Therapeutic aspects of cannabis and cannabinoids. Br. J. Psychiatry 178, 107-115 (2001).

5. Amar, M. B. Cannabinoids in medicine: a review of their therapeutic potential. J. Ethnopharmacol. 105, 1-25 (2006).

6. Whiting, P. F. et al. Cannabinoids for medical use: a systematic review and meta-analysis. Jama 313, 2456-2473 (2015).

7. Fergusson, D. M., Poulton, R., Smith, P. F. \& Boden, J. M. Cannabis and psychosis. Br. Med. J. 332, 172-176. (2006).

8. Fergusson, D. M., Hall, W., Boden, J. M. \& Horwood, L. J. Rethinking cigarette smoking, cannabis use, and psychosis. Lancet Psychiatry 2, 581-582 (2015).

9. Radhakrishnan, R., Wilkinson, S. T. \& D'Souza, D. C. Gone to pot-a review of the association between cannabis and psychosis. Front. Psychiatry 5, 54 (2014).

10. Power, R. A. et al. Genetic predisposition to schizophrenia associated with increased use of cannabis. Mol. Psychiatry 19, 1201 (2014).

11. Gage, S. H. et al. Assessing causality in associations between cannabis use and schizophrenia risk: a two-sample Mendelian randomization study. Psychological Med. 47, 971-980 (2017).

12. Horwood, L. J. et al. Cannabis and depression: an integrative data analysis of four Australasian cohorts. Drug Alcohol Depend. 126, 369-378 (2012).

13. Gibbs, M. et al. Cannabis use and mania symptoms: a systematic review and meta-analysis. J. Affect. Disord. 171, 39-47 (2015).

14. Fergusson, D. M., Boden, J. M. \& Horwood, L. J. Cannabis use and other illicit drug use: Testing the gateway hypothesis. Addiction 101, 556-569 (2006).
15. Wang, J. C., Kapoor, M. \& Goate, A. M. The genetics of substance dependence. Annu. Rev. Genom. Hum. G 13, 241-261 (2012).

16. Adkins, D. E. et al. Genome-wide meta-analysis of longitudinal alcohol consumption across youth and early adulthood. Twin Res. Hum. Genet. 18, 335-347 (2015).

17. Costello, E. J. et al. Genes, environments, and developmental research: methods for a multi-site study of early substance abuse. Twin Res. Hum. Genet. 16, 505-515 (2013).

18. Hall, W. \& Solowij, N. Adverse effects of cannabis. Lancet 352, 1611-1616 (1998).

19. Verweij, K. J. et al. Genetic and environmental influences on cannabis use initiation and problematic use: a meta-analysis of twin studies. Addiction 105, 417-430 (2010).

20. Gillespie, N. A. et al. Associations between personality disorders and cannabis use and cannabis use disorder: a population-based twin study. Addiction 113, 1488-1498 (2018).

21. Gillespie, N. A., Neale, M. C. \& Kendler, K. S. Pathways to cannabis abuse: a multi-stage model from cannabis availability, cannabis initiation and progression to abuse. Addiction 104, 430-438 (2009).

22. Pasman, J. A. et al. Genome-wide association analysis of lifetime cannabis use $(N=184,765)$ identifies new risk loci, genetic overlap with mental health, and a causal influence of schizophrenia on cannabis use. bioRxiv 234294 (2018).

23. Visscher, P. M., Brown, M. A., McCarthy, M. I. \& Yang, J. Five years of GWAS discovery. Am. J. Hum. Genet. 90, 7-24 (2012).

24. Ward, L. D. \& Kellis, M. Interpreting noncoding genetic variation in complex traits and human disease. Nat. Biotechnol. 30, 1095 (2012).

25. Fagny, M. et al. Exploring regulation in tissues with eQTL networks. Proc. Natl Acad. Sci. USA 114, E7841-E7850 (2017).

26. Jaenisch, R. \& Bird, A. Epigenetic regulation of gene expression: how the genome integrates intrinsic and environmental signals. Nat. Genet. 33, 245-254 (2003).

27. Petronis, A. Epigenetics as a unifying principle in the aetiology of complex traits and diseases. Nature 465, 721 (2010).

28. Spadafora, R. The Key Role of Epigenetics in Human Disease. N. Engl. J. Med. 379, 400 (2018).

29. Lister, R. et al. Human DNA methylomes at base resolution show widespread epigenomic differences. Nature 462, 315-322 (2009).

30. Lee, H. J., Hore, T. A. \& Reik, W. Reprogramming the methylome: erasing memory and creating diversity. Cell Stem Cell 14, 710-719 (2014).

31. Lowdon, R. F., Jang, H. S. \& Wang, T. Evolution of epigenetic regulation in vertebrate genomes. Trends Genet. 32, 269-283 (2016).

32. Lea, A. J. et al. Genome-wide quantification of the effects of DNA methylation on human gene regulation. Elife 7, e37513 (2018).

33. Moore, L. D., Le, T. \& Fan, G. DNA methylation and its basic function. Neuropsychopharmacology 38, 23 (2013).

34. Anastasiadi, D., Esteve-Codina, A. \& Piferrer, F. Consistent inverse correlation between DNA methylation of the first intron and gene expression across tissues and species. Epigenet. chromatin 11, 37 (2018).

35. Jirtle, R. L. \& Skinner, M. K. Environmental epigenomics and disease susceptibility. Nat. Rev. Genet. 8, 253-262 (2007).

36. Feil, R. \& Fraga, M. F. Epigenetics and the environment: emerging patterns and implications. Nat. Rev. Genet. 13, 97-109 (2012).

37. Garg, P., Joshi, R. S., Watson, C. \& Sharp, A. J. A survey of inter-individual variation in DNA methylation identifies environmentally responsive coregulated networks of epigenetic variation in the human genome. PLoS Genet. 14, e1007707 (2018).

38. Dominguez-Salas, P. et al. Maternal nutrition at conception modulates DNA methylation of human metastable epialleles. Nat. Commun. 5, 3746 (2014).

39. Barres, R. et al. Acute exercise remodels promoter methylation in human skeletal muscle. Cell Metab. 15, 405-411 (2012).

40. Pacis, A. et al. Bacterial infection remodels the DNA methylation landscape of human dendritic cells. Genome Res. 25, 1801-1811 (2015).

41. Rönn, T. et al. A six months exercise intervention influences the genomewide DNA methylation pattern in human adipose tissue. PLoS Genet. 9, e1003572 (2013).

42. Philibert, R. A., Plume, J. M., Gibbons, F. X., Brody, G. H. \& Beach, S. R. H. The impact of recent alcohol use on genome wide DNA methylation signatures. Front. Genet. 3, 54 (2012).

43. Philibert, R. A. et al. A pilot examination of the genome-wide DNA methylation signatures of subjects entering and exiting short-term alcohol dependence treatment programs. Epigenetics 9, 1212-1219 (2014). 
44. Zeilinger, S. et al. Tobacco smoking leads to extensive genome-wide changes in DNA methylation. PLOS ONE 8, e63812 (2013).

45. Macleod, J. et al. Psychological and social sequelae of cannabis and other illicit drug use by young people: a systematic review of longitudinal, general population studies. Lancet 363, 1579-1588 (2004).

46. McGee, R., Williams, S., Poulton, R. \& Moffitt, T. A longitudinal study of cannabis use and mental health from adolescence to early adulthood. Addiction 95, 491-503 (2000).

47. Moore, T. H. et al. Cannabis use and risk of psychotic or affective mental health outcomes: a systematic review. Lancet 370, 319-328 (2007).

48. Fergusson, D. M., Boden, J. M. \& Horwood, L. J. Psychosocial sequelae of cannabis use and implications for policy: findings from the Christchurch Health and Development Study. Soc. Psychiatry Psychiatr. Epidemiol. 50, 1317-1326 (2015).

49. Fergusson, D. M., Horwood, L. \& Swain-Campbell, N. Cannabis dependence and psychotic symptoms in young people. Psychological Med. 33, 15-21 (2003).

50. Fergusson, D. M. Is there a causal linkage between cannabis use and increased risk of psychotic symptoms? (Commentary). Addiction 105 1336-1337 (2010).

51. Fergusson, D. M., Horwood, L. J. \& Boden, J. M. Is driving under the influence of cannabis becoming a greater risk to driver safety than drink driving? Findings from a 25-year longitudinal study. Accid. Anal. Prev. 40, 1345-1350 (2008).

52. Fergusson, D. M., Horwood, L. J. \& Ridder, E. M. Tests of causal linkages between cannabis use and psychotic symptoms. Addiction 100, 354-366 (2005).

53. Fergusson, D. M., Swain-Campbell, N. R. \& Horwood, L. J. Arrests and convictions for cannabis related offences in a New Zealand birth cohort. Drug Alcohol Depend. 70, 53-63 (2003).

54. Horwood, L. J. et al. Cannabis use and educational achievement: findings from three Australasian cohort studies. Drug Alcohol Depend. 110, 247-253 (2010).

55. Association, A. P. Diagnostic and Statistical Manual of Mental Disorders Fourth edition (American Psychiatric Association, Washington, DC, 1994).

56. Pearson, J. F. et al. Increased risk of major depression by childhood abuse is not modified by CNR1 genotype. Am. J. Med. Genet. B Neuropsychiatr. Genet. 2, 224 (2013).

57. Team, R. C. R: A Language and Environment for Statistical Computing Vienna, Austria, 2019).

58. Pidsley, R. et al. Critical evaluation of the Illumina MethylationEPIC BeadChip microarray for whole-genome DNA methylation profiling. Genome Biol. 17, 208 (2016).

59. Triche, T. J. Jr, Weisenberger, D. J., Van Den Berg, D., Laird, P. W. \& Siegmund K. D. Low-level processing of Illumina Infinium DNA methylation beadarrays. Nucleic Acids Res. 41, e90-e90 (2013).

60. Jaffe, A. E. FlowSorted.Blood.450k: Illumina HumanMethylation Data on Sorted Blood Cell Populations (2019).

61. Ritchie, M. E. et al. limma powers differential expression analyses for RNAsequencing and microarray studies. Nucleic Acids Res. 43, e47-e47 (2015).

62. van Iterson, M., van Zwet, E. W., Heijmans, B. T. \& Consortium, B. Controlling bias and inflation in epigenome-and transcriptome-wide association studies using the empirical null distribution. Genome Biol. 18, 19 (2017).

63. Lawrence, $\mathbf{M}$. et al. Software for computing and annotating genomic ranges. PLoS Computational Biol. 9, e1003118 (2013).

64. Kuleshov, M. V. et al. Enrichr: a comprehensive gene set enrichment analysis web server 2016 update. Nucleic Acids Res. 44, W90-W97 (2016).

65. Ferson, M., Edwards, A., Lind, A., Milton, G. \& Hersey, P. Low natural killer-cell activity and immunoglobulin levels associated with smoking in human subjects. Int. J. Cancer 23, 603-609 (1979).

66. Mian, M. F., Lauzon, N. M., Stämpfli, M. R., Mossman, K. L. \& Ashkar, A. A. Impairment of human NK cell cytotoxic activity and cytokine release by cigarette smoke. J. Leukoc. Biol. 83, 774-784 (2008).

67. Ambatipudi, S. et al. Tobacco smoking-associated genome-wide DNA methylation changes in the EPIC study. Epigenomics 8, 599-618 (2016).

68. Breitling, L. P., Yang, R. X., Korn, B., Burwinkel, B. \& Brenner, H. Tobaccosmoking-related differential DNA methylation: $27 \mathrm{~K}$ discovery and replication. Am. J. Hum. Genet. 88, 450-457 (2011).

69. Shenker, N. S. et al. DNA methylation as a long-term biomarker of exposure to tobacco smoke. Epidemiology 24, 712-716 (2013).

70. Joehanes, R. et al. Epigenetic signatures of cigarette smoking. Circulation: Cardiovascular Genet. 9, 436-447 (2016).
71. Kilmer, B. Recreational cannabis - minimizing the health risks from legalization. N. Engl. J. Med. 376, 705-707 (2017).

72. Cerdá, M. \& Kilmer, B. Uruguay's middle-ground approach to cannabis legalization. Int. J. Drug Policy 42, 118 (2017).

73. Bifulco, M. \& Pisanti, S. Medicinal use of cannabis in Europe. EMBO Rep. 16, 130-132 (2015).

74. Wiese, B. \& Wilson-Poe, A. R. Emerging evidence for cannabis' role in opioid use disorder. Cannabis Cannabinoid Res. 3, 179-189 (2018).

75. Hall, W. Challenges in minimizing the adverse effects of cannabis use after legalization. Soc. Psychiatry Psychiatr. Epidemiol. 50, 1013-1015 (2015).

76. Boden, J. M. \& Fergusson, D. M. Cannabis law and cannabis-related harm. N.Z. Med. J. (Online) 132, 7-10 (2019).

77. Åberg, K. et al. Genomewide association study of movement-related adverse antipsychotic effects. Biol. Psychiatry 67, 279-282 (2010).

78. Platzer, $K$. et al. Haploinsufficiency of CUX1 causes nonsyndromic global developmental delay with possible catch-up development. Ann. Neurol. 84, 200-207 (2018).

79. Caspi, A. et al. Moderation of the effect of adolescent-onset cannabis use on adult psychosis by a functional polymorphism in the catechol-Omethyltransferase gene: longitudinal evidence of a gene $X$ environment interaction. Biol. Psychiatry 57, 1117-1127 (2005).

80. Henquet, C., Di Forti, M., Morrison, P., Kuepper, R. \& Murray, R. M. Geneenvironment interplay between cannabis and psychosis. Schizophrenia Bull. 34, 1111-1121 (2008).

81. Consortium, C.-D. GotP. G. Identification of risk loci with shared effects on five major psychiatric disorders: a genome-wide analysis. Lancet $\mathbf{3 8 1}$ 1371-1379 (2013).

82. Consortium, T. A. S. D. W. Go. T. P. G. Meta-analysis of GWAS of over 16,000 individuals with autism spectrum disorder highlights a novel locus at 10q24. 32 and a significant overlap with schizophrenia. Mol. Autism 8, 1-17 (2017).

83. Winham, S. J. et al. Genome-wide association study of bipolar disorder accounting for effect of body mass index identifies a new risk allele in TCF7L2. Mol. Psychiatry 19, 1010 (2014).

84. Linnér, R. K. et al. Genome-wide association analyses of risk tolerance and risky behaviors in over 1 million individuals identify hundreds of loci and shared genetic influences. Nat. Genet. 51, 245 (2019).

85. Wong, M.-L. et al. The PHF21B gene is associated with major depression and modulates the stress response. Mol. Psychiatry 22, 1015 (2017).

86. Davies, G. et al. Study of 300,486 individuals identifies 148 independent genetic loci influencing general cognitive function. Nat Commun. 9, 2098 (2018).

87. Cox, A. J. et al. Heritability and genetic association analysis of cognition in the Diabetes Heart Study. Neurobiol. Aging 35, 1958. e1953-1958 (2014).

88. Lee, J. J. et al. Gene discovery and polygenic prediction from a genome-wide association study of educational attainment in 1.1 million individuals. Nat. Genet. 50, 1112 (2018).

89. Goes, F. S. et al. Genome-wide association study of schizophrenia in Ashkenazi Jews. Am. J. Med. Genet. Part B: Neuropsychiatr. Genet. 168, 649-659 (2015).

90. Ikeda, M. et al. Genome-Wide Association Study Detected Novel Susceptibility Genes for Schizophrenia and Shared Trans-Populations/Diseases Genetic Effect. Schizophrenia Bull. 45, 824-834 (2018).

91. Lam, M. et al. Pleiotropic meta-analysis of cognition, education, and schizophrenia differentiates roles of early neurodevelopmental and adult synaptic pathways. bioRxiv, 519967 (2019).

92. Li, Z. et al. Genome-wide association analysis identifies 30 new susceptibility loci for schizophrenia. Nat. Genet. 49, 1576 (2017).

93. Periyasamy, S. et al. Association of schizophrenia risk with disordered niacin metabolism in an Indian genome-wide association study. JAMA Psychiatry 76, 1026-1034 (2019).

94. Ripke, S. et al. Biological insights from 108 schizophrenia-associated genetic loci. Nature 511, 421 (2014).

95. Herold, C. et al. Family-based association analyses of imputed genotypes reveal genome-wide significant association of Alzheimer's disease with OSBPL6, PTPRG, and PDCL3. Mol. Psychiatry 21, 1608 (2016).

96. Ripke, S. et al. Genome-wide association study identifies five new schizophrenia loci. Nat. Genet. 43, 969 (2011).

97. Karpova, A., Sanna, P. \& Behnisch, T. Involvement of multiple phosphatidylinositol 3-kinase-dependent pathways in the persistence of 
late-phase long term potentiation expression. Neuroscience 137, 833-841 (2006).

98. Sui, L., Wang, J. \& Li, B.M. Role of the phosphoinositide 3-kinase-Aktmammalian target of the rapamycin signaling pathway in long-term potentiation and trace fear conditioning memory in rat medial prefrontal cortex. Learn. Mem. 15, 762-776 (2008).

99. Kichaev, G. et al. Leveraging polygenic functional enrichment to improve GWAS power. Am. J. Hum. Genet. 104, 65-75 (2019).

100. Singh, A. et al. Cardiovascular complications of marijuana and related substances: a review. Cardiol. Ther. 7, 45-59 (2018).

101. Rezkalla, S. \& Kloner, R. A. Cardiovascular effects of marijuana. Trends Cardiovas. Med. 29, 403-407 (2018).

102. Jones, R. T. Cardiovascular system effects of marijuana. J. Clin. Pharmacol. 42 58S-63S (2002).
103. Goyal, H., Awad, H. H. \& Ghali, J. K. Role of cannabis in cardiovascular disorders. J. Thorac. Dis. 9, 2079 (2017).

104. Soto, D., Altafaj, X., Sindreu, C. \& Bayés, A. Glutamate receptor mutations in psychiatric and neurodevelopmental disorders. Communicative Integr. Biol. 7, e27887 (2014).

105. Billingsley, K. J. et al. Regulatory characterisation of the schizophreniaassociated CACNAIC proximal promoter and the potential role for the transcription factor EZH2 in schizophrenia aetiology. Schizophrenia Res. 199, 168-175 (2018).

106. Ministry of Health. Annual Update of Key Results 2017/18: New Zealand Health Survey (ed. M.o. Health) (Ministry of Health, Wellington, 2018).

107. World Health Organization. The Health and Social Effects of Nonmedical Cannabis Use (World Health Organization, 2016). 